\title{
Utility of intervention programs in the evolutional control of patients with heart failure
}

\author{
Álvaro Flamarique-Pascual', Eduardo Esteban-Zubero², Francisco José Ruiz-Ruiz' and \\ Fernando Ruiz-Laiglesia ${ }^{1}$ \\ ${ }^{1}$ Department of Medicine, Psychiatry and Dermatology; '2Department of Pharmacology and Physiology. Faculty of Medicine, Universidad de Zaragoza, \\ Zaragoza, Spain
}

\begin{abstract}
Acute heart failure (HF) is a prevalent disease with important socio-economic repercussions. Due to the aging of population, these values will increase in the coming years, so it may be useful to the implementation of intervention programs in these patients to decrease morbidity and mortality. A quasi-experimental prospective study $(n=262)$ of patients admitted at the Internal Medicine Department of the Hospital Clínico Universitario Lozano Blesa, in Zaragoza, Spain, diagnosed of HF between November 2013 and October 2014 (both dates inclusive) $(n=108)$ followed up for 1 year was performed. Within this group, a subgroup with an intensive intervention $(n=30)$ was performed. The data were compared with a historical cohort of patients admitted to the same department during the same time in the previous year (from November 2012 to October 2013) ( $n=154$ ). Statistically significant differences between groups attending to the therapeutical adherence to clinical guidelines $(p<0.011)$ were observed. Considering the intensive intervention subgroup, statistically significant differences were observed in the rate of exitus $(p<0.032)$ and survival (log rank <0.030) compared to the control group. The close monitoring of patients with $\mathrm{HF}$ improves adherence, reduces mortality and improves survival. This may result in a decline in the use of health resources, which entails significant socio-economic benefits.
\end{abstract}

KEY WORDS: Heart failure. Survival. Mortality. Therapeutic adherence. Morbidity.

\section{Introduction}

Acute heart failure $(\mathrm{HF})$ is one of the main reasons for hospital emergency department visits and is the most common cause of hospital admission in older patients. All this entails high morbidity and mortality, as well as high consumption of resources, especially those related to hospital admissions ${ }^{1,2}$. The prevalence of $\mathrm{HF}$ in the general population is estimated in around $1-3 \%$, but in patients older than 70 years, these figures have been observed to be as high as $10 \%^{2}$. Regardless of age, the risk for developing HF sometime in life has been estimated to be approximately $20 \%$ from 40 years of age on ${ }^{3}$. This is especially relevant since, currently, in developed countries, HF accounts for $2-5 \%$ of total healthcare expenditure, out of which $60-70 \%$ is directly generated by hospital admissions and readmissions, with the latter being able to reach $24-50 \%$ at 3-6 months ${ }^{4,5}$. In addition, in the USA, this condition is suffered by 6.6 million people, but this figure is estimated to likely reach 10 million by 2030 . This would imply a 50\% increase in hospitalizations, which would entail a triplication of healthcare expenditure for the treatment of this disease ${ }^{6}$. This is why care programs are being developed in order to reduce morbidity and mortality and hence the number of hospital admissions in the population older than 65 years has been able to be

\section{Correspondence:}

Álvaro Flamarique-Pascual

Date of acceptance: 27-05-2016

DOI://dx.doi.org/10.24875/GMM.M18000047
Gac Med Mex. 2017;153:537-543

Contents available at PubMed www.gacetamedicademexico.com 
reduced by $29.5 \%$, although these figures have not been correlated with a mortality decline $\mathrm{e}^{7,8}$.

In Spain, the prevalence of HF in people older than 45 years is $6.8 \%$, and it constitutes the fourth cause of mortality in the population. Furthermore, 115,895 hospital discharges were typified with this condition, with a total of over 150,000 being estimated for the year 2020. Real costs of this disease (hospital, outpatient care, pharmacy and caregiver costs) account for 4701 million Euros, with 2270 million Euros corresponding to hospital expenditure ${ }^{9}$.

The most widely used scale for HF stratification in clinical practice is the one developed by the New York Heart Association (NYHA), which describes the functional capacity a patient with HF can develop ${ }^{10}$. As regards treatment, the use of angiotensin-converting enzyme inhibitors (ACEI), angiotensin II receptor blockers (ARB-II), beta-blockers (BB) and mineralocorticoid receptor antagonists, such as aldosterone, have been shown to reduce morbidity and mortality in patients with HF. On the other hand, the use of loop diuretics is widespread in this disease as a depletive treatment to improve symptoms such as dyspnea, but there are no studies certifying a decrease in morbidity and mortality ${ }^{2}$.

The purpose of the present study is to develop a control and treatment program in our health area for patients diagnosed with HF in order to improve their quality of life by reducing readmissions, hospital emergency department frequentation and associated mortality.

\section{Method}

A quasi-experimental study was carried out in patients with a clinical diagnosis of HF who were admitted to the department of internal medicine of the Hospital Clínico Universitario Lozano Blesa, of Zaragoza, Spain, between November 2013 and October 2014, and who underwent a 1-year follow-up from admission date or until death. For data assessment, a historic cohort of patients of the same origin admitted on the same dates duing the previous year (between November 2012 and October 2013) was reviewed.

Patients were informed on the possibility to participate in the study, with their agreement being documented in the medical record. The patients corresponding to the follow-up cohort were divided into two groups according to the type of intervention they were receiving. Patients included in the high-intensity intervention modality had an appointment arranged at the internal medicine outpatient clinic one month after discharge. Periodical assessments were made (every 2 weeks), and communication with the physician was facilitated to patients via e-mail or telephone in case of any problem or incidence that might arise between visits. Patients included in the low-intensity intervention modality received a leaflet with information about their condition and general recommendations with regard to pharmacological treatment and lifestyle habits. Follow-up was carried out by their primary care physician (except for the first visit one month after hospital discharge, which took place in the department of internal medicine), and patients were not offered the possibility of contact with the physicians between appointments.

The analyzed variables (readmissions, emergency department frequentation and mortality) were registered by reviewing the medical records, from the hospital intranet and from the National Institute of Statistics. The term exitus was assigned to subjects who died during the year of follow-up, regardless of the cause of death; the term precocious exitus was assigned to subjects deceased during admission; the readmission status was assigned to subjects who were admitted to the hospital again in the course of the follow-up year; and emergency department frequentation, to patients who attended the emergency department for HF-related evaluation and were not admitted to the hospital. Hospital stay refers to the number of days patients remained admitted at the department of internal medicine.

The criteria that were followed to discern about the correct management and treatment of patients were based on the European Society of Cardiology HF guidelines ${ }^{2}$. The performance of echocardiography for the HF diagnosis was assessed as an indicator of healthcare quality. As outcome indicator, treatment adherence to the aforementioned clinical guidelines was assessed, which consists in the use of ACEI or ARB-II at any dosage, BB at any dosage and spironolactone in case of NYHA grade III-IV systolic HF at doses of 25-50 mg.

For statistical calculations, IBM SPSS Statistics v22 software was used. Quantitative variables were expressed as the mean \pm standard deviation. Normality was evaluated with Kolmogorov-Smirnoff and Shapiro-Wilk tests, and homocedasticity was verified with Levene's equality of variances test. Student's t-test was used for the comparison of quantitative variables. Mann-Whitney U-test was used as a non-parametric test for quantitative dependent variables. Categorical 
Table 1. Heart failure etiological distribution and hospital admission precipitating factors

\begin{tabular}{llll}
\hline Etiological distribution & Frequency & Precipitating factors & Frequency \\
\hline Mixed & $24 \%(n=63)$ & Unknown & $30.9 \%(n=81)$ \\
Arterial hypertension & $18.3 \%(n=48)$ & I Infection & $27.9(n=73)$ \\
Not recorded & $13.4 \%(n=35)$ & Heart arrhythmia & $10.7(n=28)$ \\
Valvular heart disease & $11.8 \%(n=31)$ & Angina & $7.3(n=19)$ \\
Cor pulmonale & $11.8 \%(n=31)$ & Anemia & $6.1(n=16)$ \\
Ischemic heart disease & $10.3 \%(n=27)$ & Lost & $5,7(n=15)$ \\
Others & $3.8 \%(n=10)$ & Chronic obstructive pulmonary disease & $3.8(n=10)$ \\
Dilated cardiomyopathy & $3.4 \%(n=9)$ & Wrong treatment & $2.7(n=7)$ \\
Diabetes mellitus & $1.5 \%(n=4)$ & Other & $2.3(n=6)$ \\
Restrictive myocardiopathy & $0.8 \%(n=2)$ & Arterial hypertension & $1.1(n=3)$ \\
Hypertrophic & $0.4 \%(n=1)$ & Pulmonary thromboembolism & $1.1(n=3)$ \\
myocardiopathy & & Hyperthyroidism & Total \\
Hyperthyroidism & $0.4 \%(n=1)$ & $100 \%(n=262)$ & $0.4(n=1)$ \\
Total & & $100 \%(n=262)$ \\
\hline
\end{tabular}

variables were analyzed with the chi-square test. The Kaplan-Meier method was used for survival analysis, with the different survival curves being compared with the log-rank test. Differences with a p-value $<0.05$ were considered to be significant.

\section{Results}

Data were collected from 262 patients, out of which 108 belonged to the intervention group. Within this intervention group, there were two types of patients: those who were strictly followed at internal medicine outpatient visits (high-intensity follow-up group, $\mathrm{n}=$ 30) and those with low-intensity follow-up (general recommendations, $\mathrm{n}=78$ ). Mean age was $78.27 \pm$ 8.30 years. Among the study subjects, $54.6 \%$ were females $(n=143)$ and $45.4 \%$ were males $(n=119)$. HF most common etiology in our series was mixed, which included patients with at least two concomitant conditions, generally arterial hypertension and diabetes mellitus. Arterial hypertension was the most relevant single etiology ( $18.3 \%$ of cases). No statistically significant differences were appreciated in both groups' characteristics $(p<0.05)$. Data on the HF etiology is shown in table 1.

With regard to the distribution of admissions over the year, the highest number was found to correspond to winter months (Fig. 1). Most common precipitating factor was infection (27.9\% of cases), although it should be noted that the cause was not known in a high proportion of cases (30.9\%).

\section{Quality indicators' analysis}

The performance of echocardiography was used as an indicator of quality of the process since, according to the clinical guidelines, it has to be performed in all HF-diagnosed patients in order to assess heart dysfunction ${ }^{2}$. In the control group, it was performed in $47.9 \%$ of patients, whereas in the intervention group it was performed in $67 \%$, with statistically significant differences being therefore appreciated $(p<0.05)$. Nevertheless, neither group reached the quality standards for HF diagnosis. There were no differences between both cohorts in terms of distribution according to the type of dysfunction (systolic or diastolic) ( $p$ $>0.05$ ). In the control group, $60.3 \%$ of subjects had diastolic-type and $39.7 \%$ systolic-type $\mathrm{HF}$; in the intervention group, the percentages were $61.5 \%$ and $38.5 \%$, respectively.

\section{Outcome indicators analysis}

Statistically significant differences were observed for the use of $\mathrm{BB}$ in the intervention cohort in comparison with the control group $(p<0.001)$. No statistically significant differences were found for the use of ACEl, although an increased use in the intervention group in 


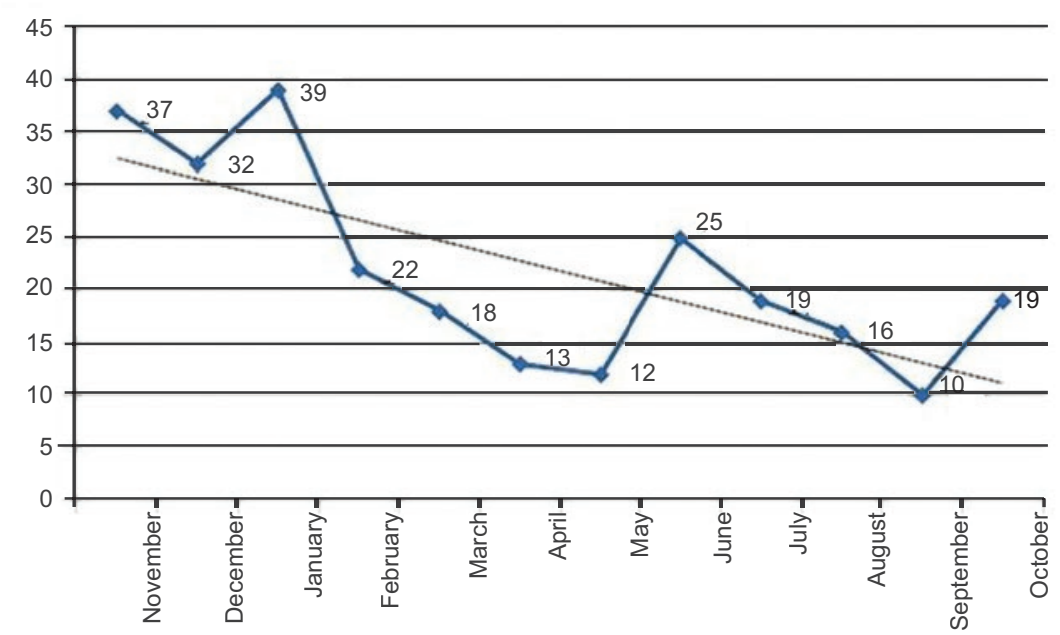

Figure 1. Number of hospital admissions according to the month of the year. The values correspond to the subjects included in the intervention group $(n=262)$.

Tabla 2. Pharmacological groups used and treatment adherence.

\begin{tabular}{lccc}
\hline Treatment & Control group & Intervention group & Total \\
\hline Beta-blocker & Yes: $22 \%(n=29)$ & Yes: $42.1 \%(n=40)^{*}$ & Yes: $30.4 \%(n=69)$ \\
& No: $78 \%(n=103)$ & No: $57.9 \%(n=55)$ & No: $69.6 \%(n=158)$ \\
Angiotensin-converting enzyme inhibitors & Yes: $52.3 \%(n=69)$ & Yes: $64.9 \%(n=61)$ & Yes: $57.5 \%(n=130)$ \\
& No: $47.7 \%(n=63)$ & No: $35.1 \%(n=33)$ & No: $42.5 \%(n=96)$ \\
Angiotensin II receptor blockers & Yes: $16.7 \%(n=22)$ & Yes: $15.8 \%(n=15)$ & Yes: $16.3 \%(n=37)$ \\
& No: $83.3 \%(n=110)$ & No: $84.2 \%(n=80)$ & No: $83.3 \%(n=190)$ \\
Treatment adherence & Yes: $39.3 \%(n=53)$ & Yes: $56.7 \%(n=55)^{*}$ & Yes: $46.6 \%(n=108)$ \\
& No: $60.7 \%(n=82)$ & No: $43.3 \%(n=42)$ & No: $53.4 \%(n=124)$ \\
\hline${ }_{*}<0.05$ & & &
\end{tabular}

${ }^{{ }^{p} \mathrm{p}<0.05}$

comparison with the control group should be highlighted (64.9 and $52.3 \%$, respectively). ARB-II prescription was similar in both cohorts $(15.8 \%$ on prospective follow-up and $16.7 \%$ in the retrospective group). With regard to adherence to clinical guidelines, defined as compliance by clinicians with currently valid scientific recommendations with regard to a disease (which in this case translates into the use of ACEI/ARB-II, BB and mineralocorticoid receptor antagonists to reduce morbidity and mortality in HF), statistically significant differences were found between the intervention and the follow-up groups $(p<0.011)$ (Table 2).

As for the exitus variable, although a higher percentage of deaths are appreciated in the control group $(22.5 \%)$ than in the intervention group (19.4\%), no statistically significant differences were observed between both $(p>0.05)$ (Table 3). Subjects classified as lost in this variable with regard to total number of patients correspond to precocious exitus, with no significant differences found between both groups. The high-intensity subgroup shows statistically significant differences in the rate of exitus once precocious exitus are discarded, in comparison with the control group (0 and $15.5 \%$, respectively; $p<0.032$ ) (Fig. 2). Similar results were observed in the survival analysis throughout the study period, without differences being appreciated between the control and intervention groups (log-rank: 0.708), although differences were found in the high-intensity follow-up subgroup in comparison with the historic cohort (log-rank: < 0.030) (Fig. 3).

As regards the number of readmissions, the mean in both groups (prospective and retrospective) was very similar, with no differences being found within the intervention subgroups (Table 3).

When emergency department frequentation was analyzed, a slightly higher mean was observed in the intervention group in comparison with the control group, with no statistically significant differences being observed. No differences were observed between the intervention subgroups as well (Table 1). 


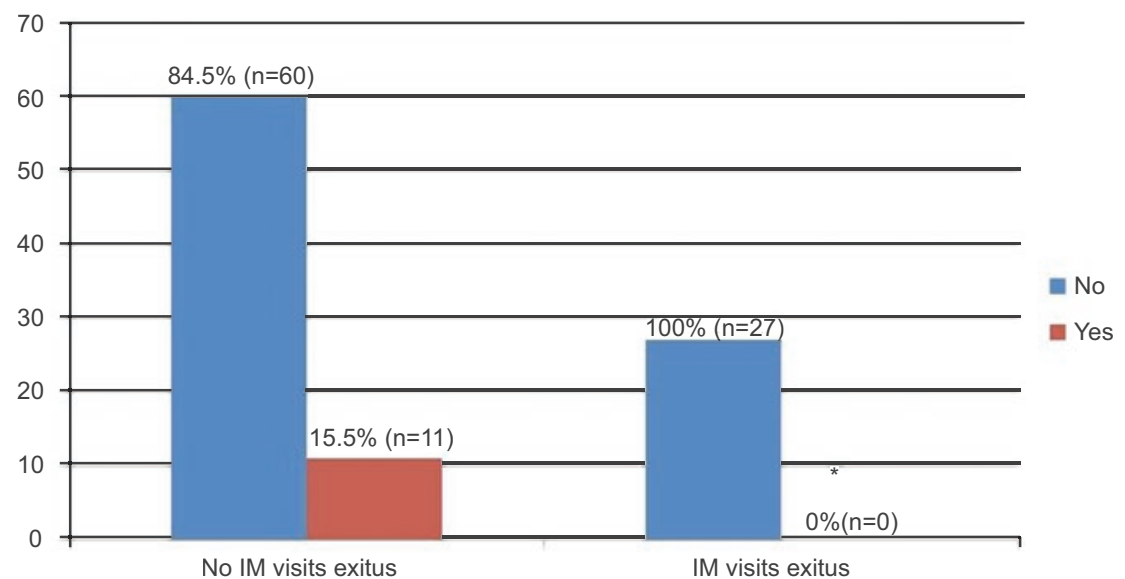

Figure 2. Intervention group exitus according to subgroups (higher intensity intervention with follow-up at internal medicine department outpatient clinic visits or lower intensity intervention with general recommendations by the same department. When the subgroup of patients examined at outpatient clinic visits is compared with the rest of the follow-up group, statistically significant differences are observed $(p>0.032)$.
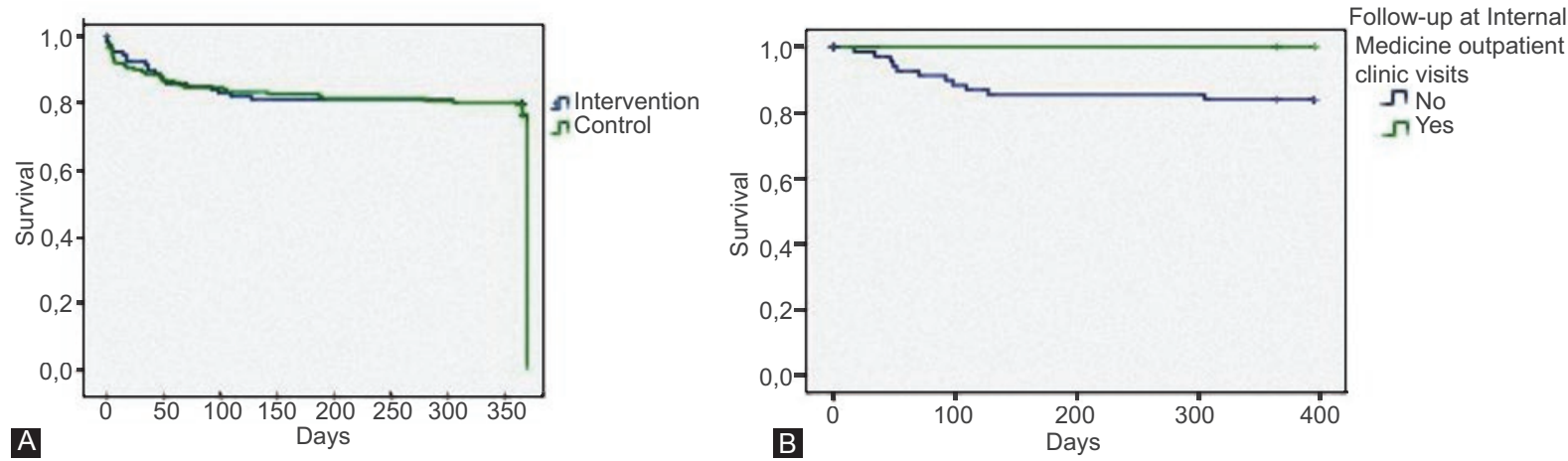

Figure 3. A: survival curve over the time analyzed in the study, where the control and intervention groups are compared without the type of intervention (high or low intensity being differentiated. No statistically significant differences were appreciated between both (log-rank: 0.708). B: statistically significant differences were observed within the intervention group in high-intensity group (follow-up at internal medicine outpatient clinic visits) and in low intensity group (no follow-up at internal medicine outpatient visits) survival (log-rank: $<0.030$ ).

No statistically significant differences were shown in hospital stay between both groups, with it being slightly longer in the intervention group, with 11.70 days' mean stay (Table 1).

\section{Discussion}

The most common cause of cardiac decompensation was infectious, followed by heart conditions such as ischemic heart disease and heart arrhythmia, which is consistent with previous studies ${ }^{11}$. Looking at both groups, the limited performance of the echocardiograpihic study in our setting should be highlighted, which may be due to its delayed performance at our institution. This problem is common in other studies reviewed in the literature ${ }^{12}$.

As regards treatment, a quite acceptable adherence to the evaluated therapeutic recommendation guidelines is appreciated ${ }^{2}$. Of note, there was a statistically significant difference in the use of $\mathrm{BB}$, with higher rates being observed in the intervention group than in the control group. This data is elevant, since several studies have observed a prognostic improvement with the use of these drugs in asymptomatic patients with left ventricle systolic dysfunction, thus preventing progression to symptomatic HF, decreasing and preventing ventricular remodeling and hence increasing survival $^{13}$. Higher treatment adherence in HF patients with stricter follow-up has been observed in previous studies, especially with regard to an increase in the use of $\mathrm{BB}^{14-16}$.

Our work also reveals a decline in the exitus rate and a survival increase in the high-intensity intervention group (with follow-up at outpatient clinic visits), in comparison with the control group. These data are consistent with the reviewed literature, with decreases in the mortality rate being appreciated one year after the HF diagnosis with increased treatment adherence 
Tabla 3. Rate of exitus, precocious exitus, hospital readmissions, emergency department frequentation and hospital stay according to their follow-up with high to low intensity intervention

\begin{tabular}{|c|c|c|c|c|}
\hline $\begin{array}{l}\text { Analyzed } \\
\text { variable }\end{array}$ & Control group & Intervention group & Intervention subgroup & Total \\
\hline \multirow[t]{2}{*}{ Exitus } & Yes: $22.5 \%(n=34)$ & Yes: $19.4 \%(n=21)$ & High intensity: $0 \%(n=0)^{*}$ & Yes: $21.2 \%(n=55)$ \\
\hline & No: $77.5 \%(n=117)$ & No: $80.6 \%(n=87)$ & Low intensity: $15.5 \%(n=11)$ & No: $78.8 \%(n=204)$ \\
\hline \multirow{2}{*}{$\begin{array}{l}\text { Precocious } \\
\text { Exitus }\end{array}$} & Yes: $10 \%(n=15)$ & Yes: $9.3 \%(n=10)$ & & Yes: $9.7 \%(n=25)$ \\
\hline & No: $90 \%(n=135)$ & No: $90.7 \%(n=98)$ & & No: $90.3 \%(n=233)$ \\
\hline \multirow{2}{*}{$\begin{array}{l}\text { Hospital } \\
\text { readmissions }\end{array}$} & $0.67 \pm 0.083(n=134)$ & $0.63 \pm 0.104(n=98)$ & High intensity: $0.63 \pm 0.116(n=27)$ & \\
\hline & & & Low intensity: $0.63 \pm 0.227(n=71)$ & \\
\hline \multirow{2}{*}{$\begin{array}{l}\text { Emergency } \\
\text { department } \\
\text { frequentation }\end{array}$} & $0.22 \pm 0.040(n=206)$ & $0.26 \pm 0.114(n=27)$ & High intensity: $0.26 \pm 0.114(n=27)$ & \\
\hline & & & Low intensity: $0.21 \pm 0.063(n=71)$ & \\
\hline Hospital stay & $11.27 \pm 0.502$ & $11.70 \pm 0.692$ & & \\
\hline
\end{tabular}

and interventions on lifestyle habits such as physical exercise and hygienic-dietary education ${ }^{14}$. With this type of intervention, improvements in quality of life have also been observed ${ }^{17}$, even with workshop-type interventions for better knowledge of the disease and maintenance of healthy lifestyle habits without pharmacological treatment being modified ${ }^{18}$. Similar studies with similar populations to ours have also been performed over time ${ }^{16,19}$. In our setting, similar results are observed in projects carried out jointly between hospital-based and primary care settings, with lower decreases observed in the mortality rate than in our study, but significantly reducing the number of readmissions ${ }^{19,20}$. There are even some studies referring a direct relationship between the number of readmissions and higher use of $\mathrm{BB}^{21}$. This differs from our work, although this might be due to differences in the samples and follow-up limited duration.

Interventional studies do not only examine secondary intervention, as in our case, but they can also assess primary interventions. It is important for awareness on healthy lifestyle habits to be emphasized in society, as well as on adequate control of cardiovascular risk factors (arterial hypertension, dyslipidemia, obesity, etc.). Tighter primary prevention has been observed to entail lower risk for the development of $\mathrm{HF}^{22}$. In said study, a lower rate of HF development was observed in hypertensive subjects with healthy lifestyle habits without pharmacological treatment in comparison with pharmacologically-treated subjects without lifestyle modification. This statement is consistent with studies where higher risk for HF and poorer prognosis are observed in those patients with elevated body mass index $(\mathrm{BMI})^{23}$, even at middle ages of life ${ }^{24}$. Less tangible variables, such as depression and anxiety, have also been observed to cause an increase in the risk for the development of $\mathrm{HF}^{25}$.

Our study does not assess economic issues, although it does it indirectly by assessing the emergency department frequentation rate, the number of readmissions and mean hospital stay. It should be noted that our results may be biased by the limited continuity of follow-up in time, which can render applied measures not reflecting on patients. Owing to this, we didn't appreciate differences for these variables between the control and the intervention group, which differs from studies where better health control is observed in intervention groups, and with lower healthcare $\operatorname{cost}^{15}$. This expenditure is based not only on administered medication, but also on indirect costs (medical and nursing personnel, etc.) resulting from the use of primary care and emergency services. This variable is important, since studies assessing the economic impact HF will have by the year 2025 due to population aging, estimate it at a mean of $14.63 \%$ more per patient. This, extrapolated to the increase in the number of readmissions should current mean tendency continue, would entail an increase in healthcare expenditure from $€ 1,271,281,638$ to $€ 1,796,803,008$ per year in developed countries ${ }^{26}$. Mean hospital stay rates observed in our patients are similar or even slightly lower than those reported in the literature ${ }^{27}$.

In conclusion, HF-affected patients' tighter follow-up improves treatment adherence, decreases mortality 
and improves survival. Even when our results do not reflect it, most times it entails a decrease in the use of healthcare resources, and thus it implies an important benefit both for the patient and the economy. This is why continued care programs for HF-affected patients should be jointly developed at hospital-based and primary care settings. This should be applicable to primary prevention as well.

\section{References}

1. Llorens P, Miró O, Martín Sánchez FJ, et al. Manejo de la insuficiencia cardiaca aguda en los servicios de urgencias, emergencias y unidades adscritas. Documento de consenso del Grupo de Insuficiencia Cardiaca Aguda de la Sociedad Española de Medicina de Urgencias y Emergencias (ICA-SEMES). Emergencias. 2011:23:119-39.

2. McMurray JJ, Adamopoulos S, Anker SD, et al. ESC Guidelines for the diagnosis and treatment of acute and chronic heart failure 2012: The Task Force for the Diagnosis and Treatment of Acute and Chronic Heart Failure 2012 of the European Society of Cardiology. Developed in collaboration with the Heart Failure Association (HFA) of the ESC. Eur Heart J. 2012;33:1787-847.

3. Ammar KA, Jacobsen SJ, Mahoney DW, et al. Prevalence and prognostic significance of heart failure stages. Application of the American College of Cardiology/American Heart Association Heart Failure staging criteria in the community. Circulation. 2007:115:1563-70.

4. Roger VL. The heart failure epidemic. Int J Environ Res Public Health. 2010;7:1807-30.

5. Setoguchi S, Stevenson LW. Hospitalizations in patients with heart failure: who and why. J Am Coll Cardiol. 2009;54:1703-5.

6. Go AS, Mozaffarian D, Roger VL, et al.; American Heart Association Statistics Committee and Stroke Statistics Subcommittee. Heart disease and stroke statistics. 2013 Update. A report from the American Heart Association. Circulation. 2013;127:e6-245.

7. Chen J, Normand SLT, Wang Y, et al. National and regional trends in heart failure hospitalization and mortality rates for Medicare beneficiaries, 1998-2008. JAMA. 2011;306:1669-78.

8. Gheorghiade M, Braunwald E. Hospitalizations for heart failure in the United States - a sign of hope. JAMA. 2011;306:1705-6.

9. Montes-Santiago J, Arévalo Lorido JC, Cerqueiro González JM. Epidemiología de la insuficiencia cardiaca aguda. Med Clin (Barc). 2014;142(Supl 1):3-8

10. The Criteria Committee of the New York Heart Association. Nomenclature and criteria for diagnosis of diseases of the heart and great vessels. 9th ed. Boston: Little, Brown \& Co; 1994. p. 253-6.
11. Blecker S, Paul M, Taksler G, et al. Heart failure-associated hospitalizations in the United States. J Am Coll Cardiol. 2013;61:1259-67.

12. Ambrosy AP, Fonarow GC, Butler J, et al. The global health and economic burden of hospitalizations for heart failure: lessons learned from hospitalized heart failure registries. J Am Coll Cardiol. 2014;63:1123-33.

13. Klapholz M. Beta-blocker use for the stages of heart failure. Mayo Clin Proc. 2009;84:718-29.

14. Nakano A, Johnsen SP, Frederiksen BL, et al. Trends in quality of care among patients with incident heart failure in Denmark 2003-2010: a nationwide cohort study. BMC Health Serv Res. 2013;13:391.

15. Agvall $B$, Paulsson $T$, Foldevi $M$, et al. Resource use and cost implications of implementing a heart failure program for patients with systolic heart failure in Swedish primary health care. Int J Cardiol. 2014;176:731-8.

16. von Scheidt W, Zugck C, Pauschinger M, et al. Characteristics, management modalities and outcome in chronic systolic heart failure patients treated in tertiary care centers: results from the EVIdence based TreAtment in Heart Failure (EVITA-HF) registry. Clin Res Cardiol. 2014;103: 1006-14.

17. Hole T, Grundtvig M, Gullestad L, et al. Improved quality of life in Norwegian heart failure patients after follow-up in outpatient heart failure clinics: results from the Norwegian Heart Failure Registry. Eur $\mathrm{J}$ Heart Fail. 2010;12:1247-52.

18. Smeulders ES, van Haastregt JC, Ambergen T, et al. The impact of a self-management group programme on health behaviour and healthcare utilization among congestive heart failure patients. Eur $\mathrm{J}$ Heart Fail. 2009;11:609-16.

19. Agvall $B$, Alehagen $U$, Dahlström $U$. The benefits of using a heart failure management programme in Swedish primary healthcare. Eur $\mathrm{J}$ Heart Fail. 2013;15:228-36.

20. Comín-Colet J, Verdú-Rotellar JM, Vela E, et al. Efficacy of an integrated hospital-primary care program for heart failure: a population-based analysis of 56,742 patients. Rev Esp Cardiol. 2014;67:283-93.

21. Hebert K, Quevedo HC, Gogichaishvili I, et al. Feasibility of a heart failure disease management program in eastern Europe: Tbilisi, Georgia. Circ Heart Fail. 2011;4:763-9.

22. Wang $Y$, Tuomilehto $J$, Jousilahti $P$, et al. Healthy lifestyle status, antihypertensive treatment and the risk of heart failure among Finnish men and women. J Hypertens. 2013;31:2158-64.

23. Puig T, Ferrero-Gregori A, Roig E, et al. Prognostic value of body mass index and waist circumference in patients with chronic heart failure (Spanish REDINSCOR Registry). Rev Esp Cardiol. 2014;67:101-6.

24. Borné $Y$, Hedblad B, Essén B, et al. Anthropometric measures in relation to risk of heart failure hospitalization: a Swedish population-based cohort study. Eur J Public Health. 2014;24:215-20.

25. Rod NH, Andersen I, Prescott E. Psychosocial risk factors and heart failure hospitalization: a prospective cohort study. Am J Epidemiol. 2011; 174:672-80.

26. Schmidt S, Hendricks V, Griebenow R, et al. Demographic change and its impact on the health-care budget for heart failure inpatients in Germany during 1995-2025. Herz. 2013;38:862-7.

27. Sugrue A, Smyth $Y$, Hennessy $S$, et al. Temporal trends in hospitalisations for heart failure. Ir Med J. 2015;108:207-9. 